\title{
Numerical Approximation of the SEIR Epidemic Model Using the Variational iteration Orthogonal Collocation method and Mamadu-Njoseh Polynomials
}

Ebimene J. Mamadu 1,*, Ignatius N. Njoseh ${ }^{2}$, Newton I. Okposo ${ }^{3}$, Herietta I. Ojarikre4, John N. Igabari ${ }^{5}$, Peter E. Ezimadu' ${ }^{6}$, Marcus I. Ossaiugbo 7 , and Abel M. Jonathan ${ }^{8}$

1 Affiliation 1; emamadu@delsu.edu.ng

2 Affiliation 2; njoseh@delsu.edu.ng

Affilia tion 3; niokposo@delsu.edu.ng

Affiliation 4; ojarikreify@delsu.edu.ng

Affiliation 5; in igabari@delsu.edu.ng

Affiliation 6; ezimadu@delsu.edu.ng

Affiliation 7; ossaiugbo@delsu.edu.ng

Affiliation 8; amjonathan@delsu.edu.ng

* Correspondence: emamadu@delsu.edu.ng; Tel.: +2348062650313

\begin{abstract}
In this paper, we proposed the numerical method called the variational iteration orthogonal collocation method (VIOCM), for the approximate solution of the deadly Corona virus model using Mamadu-Njoseh polynomials as basis functions. The proposed method is an elegant mixture of the variational iteration method (VIM) and the orthogonal collocation method (OCM). It was observed that the proposed method converges rapidly to the exact solution even as $\mathrm{N}$ increases. This suggests that the use of orthogonal polynomials as trial functions for the SEIR model is indeed an effective approximant as it produces the analytic solution at just few iterations. Resulting numerical evidences were compared with the standard variational iteration method as available in literature. All computational framew orks were executed with MAPLE softw are.
\end{abstract}

Keywords: Orthogonal collocation; Variational iteration method; Mamadu-Njoseh polynomials; differential equations; Corona Virus;SEIR model.

\footnotetext{
1. Introduction

Coronavirus disease, COVID-19, otherwise known as severe acute respiratory syndrome coronavirus 2, SARS-CoV-2, is a global threat to public health [1]. In 2019, Wuhan, China was identified as the epicentre of the SARS-CoV-2 outbreak [2-3]. The w orld health organisation (WHO) has declared COVID-19 a pandemic on March 11th, 2020 and has recorded over five hundred thousand disease-induced deaths globally since the disease outbreak [4]. COVID-19 has brought great challenges and hardship to the lives of millions of people and could have a far reaching and negative impact on the global economy if effective control measures are not put in place. SARS-CoV-2 is a communicable disease and attacks the lower respiratory system and causes damage to the heart, liver, kidney, gastrointestinal system and central nervous system, and this could result to multiple organ failure [5-9].
} 
Mathematical models have been used by scholars to study the transmission pattern and control strategies of COVID-19 and also for calculating the basic reproduction numbers [10-15]. SEIR models which are based on the division of the population under study into the susceptible, exposed, infectious and recovered compartments, have been used to simulate the effects of SARS-CoV-2 and forecast the COVID-19 outbreak [16-18]. The author [17] extended the model presented by [18] for the control of SARS to the control of COVID-19. The study ascertained the effectiveness of public health education, quarantine and isolation in reducing the COVID-19 infection and the time taken to achieve that. Pontryagin's maximum principle has been employed in the optimal control of many infectious diseases including COV ID-19 [17, 19]. Also, the author [17] reveals that optimal control is the generalisation of the optimization theory's classical calculus of variation which has to do with minimizing the cost functional, obtaining the Hamiltonian of the control model and applying the Pontryagin's maximum principle. It is clear from the foregoing that there are existing deterministic models for the analysis and control of the SARS-CoV-2, which can be solved either analytically or numerically. However, existing analytic methods such as, the Elzaki transform method, Laplace transform method, d-expansion method etc, requires complex mathematical configurations such as linearization, perturbation, quasi-linearization, and weak assumptions, which is often time consuming and challenging to undertake [20-28].

Over the years, numerical techniques have been explored for seeking the approximate solution of many mathematical models that exist in science and technology. This is due to the unfavourable situations encounters in executing most of the analytics methods. Basic unique characteristic of numerical methods lies in their ability to approximate the analytic solution of the model in a convergent series solution. Population existing numerical methods for solving many real-life problems can be seen in [29-36] and the references therein.

In this paper, we proposed an efficient and reliable numerical procedure called the " variational iteration orthogonal collocation method (VIOCM)" for the numerical approximation of SEIR (COV ID-19) model. The proposed method is an elegant mixture of the variational iteration method (VIM) and the orthogonal collocation method (OCM) employing the Mamadu-Njoseh orthogonal polynomials as basis functions in the approximation of the analytic solution of the SEIR model [37-38].

The standard variational iteration method (VIM) was first proposed by He [39]. The VIM in recent years has been explored by many researchers to solve both linear and nonlinear problems due to its simplicity of application, less computational time, and rapid rate of convergence. The method involves the construction of correction functional for the existing problem, the use of variational theory and restricted variables [40]. The method is also independent of small parameter variation in the differential equation and present its solution as a sequence of series solution.

The use of orthogonal polynomials for the solution of differential equations was first uncovered in the 1930s [41]. An equation in a given closed interval possess a collocation solution in a given collocation space if it satisfies the cardinality of the dimension of that collocation space. How ever, it 
is an orthogonal collocation if the set of collocation points is at the zeros of orthogon al collocation [42].

Since the outbreak of the novel coronavirus, many numerical schemes have been put forward by different researchers worldwide to study, analyze and find a solution for the Coronavirus model [ 26, 43-44]. However, the adoption of orthogonal polynomials as trial functions for seeking the approximate solution of the Coronavirus model has not been explored. Therefore, it is the aim of this article to seek the approximate solution of the SEIR (COVID-19) model using the Mamadu-Njoseh polynomials as basis functions via the proposed method VIOCM. The choice of these polynomials is the fact that their existence is new and possess same rate of convergence as that of Chebyshev orthogonal polynomials [45-47].

\section{Materials and Methods}

\subsection{Orthogonal polynomials}

Let $[45-46]$

$\int_{-1}^{1} w(x) \varphi_{n}(x) \varphi_{m}(x) d x= \begin{cases}0, & n \neq m \\ 1_{v} & n=m^{*}\end{cases}$

where $w(x)$ is a positive and continuous weight function on $[-1,1]$ such that the moment

$\mu=\int_{-1}^{1} w(x) x^{n} d x, n=0,1,2, \ldots$

exists. Then the inner product of $\varphi_{n}(x)$ and $\varphi_{m}(x)$ denoted as $\left\langle\varphi_{n}(x), \varphi_{m}(x)\right\rangle$ is orthogonal if

$$
\left\langle\varphi_{m}(x), \varphi_{n}(x)\right\rangle=0, \quad m \neq n, \quad x \in[-1,1]
$$

\subsubsection{Mamadu-Njoseh Polynomials, $\varphi_{n}(\boldsymbol{x})$}

These are orthogonal polynomials constructed via the weight function $w(x)=1+x^{2}, x \in[-1,1]$ using the following properties [45]:

i. $\varphi_{n}(x)=\sum_{i=0}^{n} a_{i} x^{i}$

ii. $\left\langle\varphi_{n}(x), \quad \varphi_{m}(x)\right\rangle=0, \quad m \neq n_{x} \quad x \in[-1,1]$

iii. $\varphi_{n}(1)=1$.

Thus, the first seven Mamadu-Njoseh polynomials are given as follow s:

$\varphi_{0}(x)=1$

$\varphi_{1}(x)=x$ 


$$
\begin{aligned}
& \varphi_{2}(x)=\frac{1}{3}\left(5 x^{2}-2\right) \\
& \varphi_{a}(x)=\frac{1}{5}\left(14 x^{a}-9 x\right) \\
& \varphi_{4}(x)=\frac{1}{648}\left(333-2898 x^{2}+3213 x^{4}\right) \\
& \varphi_{5}(x)=\frac{1}{136}\left(325 x-1410 x^{3}+1221 x^{5}\right) \\
& \varphi_{6}(x)=\frac{1}{1064}\left(-460+8685 x^{2}-24750 x^{4}+17589 x^{6}\right)
\end{aligned}
$$

The graphs of these polynomials (up to $\mathrm{n}=5$ ) are shown below:

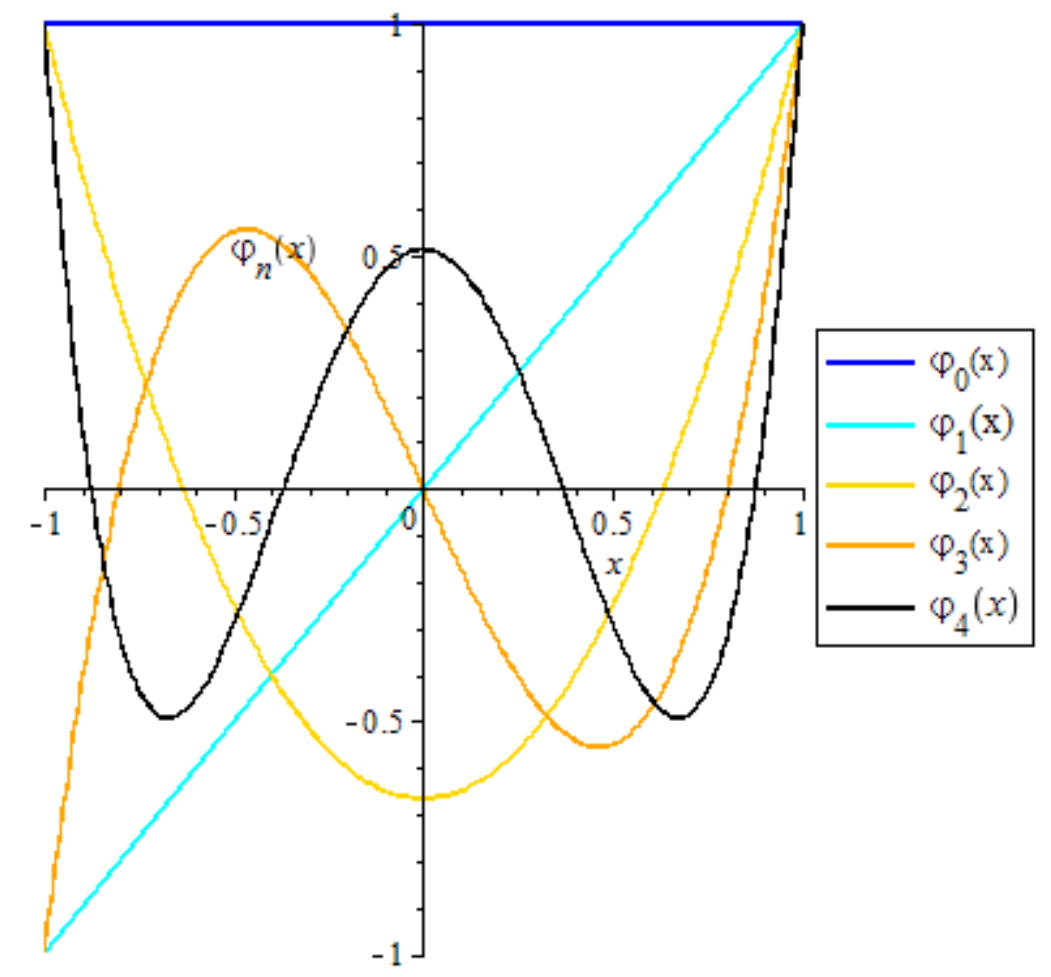

Figure 1: Mamadu-Njoseh Polynomials

\subsection{SEIR Model}

In epidemiology, the model is defined via the non-linear systems of differential equations below [37 $-38]$

$$
\begin{aligned}
& S(t)+a S(t) I(t)=0 \\
& E(t)-a S(t) I(t)+b E(t)=0 \\
& I(t)-b E(t)+c I(t)=0 \\
& R(t)-c I(t)=0
\end{aligned}
$$

where individuals of the population that are susceptible, exposed, infectious and recovered are denoted by S, E, I and R respectively. Also, a, b and c are positive constants.

Below is a systematic diagram for the various compartments of the SEIR model in the transmission among individuals in the populations.

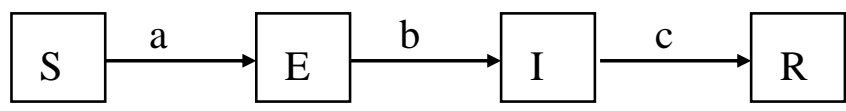




\section{Figure 2: SEIR transmission model}

\section{Interpretation of the SEIR model (CORONA VIRUS MODEL)}

Below is a precise interpretation of the SEIR model arising from (4):

i. $\quad S(t)=\frac{a}{N_{0}} S(t) I(t)-\frac{k}{N_{0}} S(t)+\left(\beta_{1}+\beta_{2}\right)-\frac{\left(\alpha_{1}+\alpha_{2}\right)}{N_{0}} S(t)+g N_{0}(t)-\tau S(t)$

where:

$S(t) \equiv \frac{d S(t)}{d t}=$ rate of change of susceptible individuals in the population.

$\frac{a}{N_{0}} S(t) I(t)=$ proportion of the population that is susceptible $\times$ average value of the population infected by a carrier (infectious individual) over the entire duration of

infection $\times$ population infected by infectious individual.
$\frac{k}{N_{0}} S(t)=$ fraction of the population that is susceptible source of infectious animal.

$\left(\beta_{1}+\beta_{2}=\right.$ entrance of travellers.

$\frac{\left(\alpha_{1}+\alpha_{2}\right)}{N_{0}} S(t)$ of susceptible persons. $g N_{0}(t)=$ birth rate due to natural occurrence multiplying the entire population.

$\tau S(t)=$ rate of death occurrence of susceptible individual multiplying the average number of susceptible individuals.

ii. $\quad E(t)=\frac{a}{N_{0}} S(t) l(t)-\frac{k}{N_{0}} S(t)-b E(t)-\frac{\left(\alpha_{1}+\alpha_{2}\right)}{N_{0}} E(t)-\tau E(t)-c E(t)$

where:

$E(t)=\frac{d E(t)}{d t}=$ rate of change of exposed individuals in the population.

$b E(t)=$ average number of persons exposed over the latency period.

$\frac{\left(a_{1}+\alpha_{2}\right)}{N_{0}} E(t)=$ proportion of population in percentage travelling out multiplying the population of
exposed persons.

$\tau E(t)=$ rate of death occurrence of exposed persons (individuals) multiplying the average number of exposed persons.

$c E(t)=$ therapy and testing rate multiplying the number of exposed individuals.

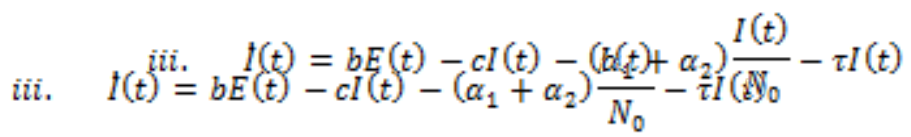

where: 
$I(t)=$ rate of change of infected persons in the population.

$b E(t)=$ average number of persons exposed over the latency period.

$c(t)=$ average number of infected persons in the duration of the infection.

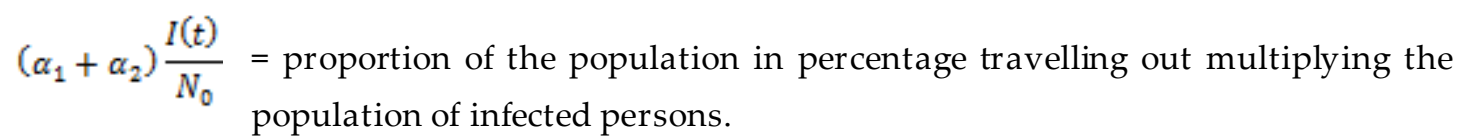

$\tau l(t)=$ rate of death of infected persons multiplying the average number of infected persons.

iv. $\quad R(t)=c l(t)-\tau R(t)+c E(t)$

where:

$\not R(t)$ = rate of change of recovered persons in the population.

$c I(t)=$ average number of infected persons in the duration of the infection.

$\tau R(t)=$ rate of death of infected persons multiplying the average number of recovered persons.

$c E(t)=$ therapy and testing rate multiplyinh the number of recovered individuals.

Thus, the systematic transmission of the various compartments of the novel coronavirus model (model) is now expressed by the following first order differential systems:

$\left.\begin{array}{l}S(t)=\frac{a}{N_{0}} S(t) I(t)-\frac{K}{N_{0}} S(t)+\left(\beta_{1}+\beta_{2}\right)-\frac{\left(\alpha_{1}+\alpha_{2}\right)}{N_{0}} S(t)+g N_{0}(t)-\tau S(t) \\ E(t)=\frac{a}{N_{0}} S(t) I(t)-\frac{K}{N_{0}} S(t)-b E(t)-\frac{\left(\alpha_{1}+\alpha_{2}\right)}{N_{0}} E(t)-\tau E(t)-\rho E(t) \\ I(t)=b E(t)-c l(t)-\left(\alpha_{1}+\alpha_{2}\right) \frac{I(t)}{N_{0}}-\tau I(t) \\ R(t)=c l(t)-\tau R(t)+\rho E(t)\end{array}\right\}$.

subject to the following constraints:

$S(0)=k_{0}, \quad E(0)=k_{1}, \quad I(0)=k_{2}, \quad R(0)=k_{a}$ 
Table 1: Definition of Parameters

\begin{tabular}{|c|c|}
\hline Parameter & Description \\
\hline$N_{0}$ & Entire population, \\
\hline$a$ & Average value of the population infected by an infectious individual \\
\hline & over the entire duration of infection. \\
\hline$b$ & Average number of persons exposed over the latency period. \\
\hline$c$ & Average number of infected persons in the duration of the infection. \\
\hline$\alpha_{1}$ & Daily number of international air inbound travellers. \\
\hline$\alpha_{2}$ & Daily number of domestic air inbound travellers. \\
\hline$\beta_{1}$ & Daily number of international air outbound travellers. \\
\hline$\beta_{2}$ & Daily number of domestic air outbound travellers. \\
\hline$\tau$ & Rate of death occurrence. \\
\hline$g$ & Birth rate occurrence due to natural occurrence. \\
\hline$k$ & Scenario of baseline force of infection. \\
\hline$\rho$ & Therapy and testing rate of the population. \\
\hline
\end{tabular}

\subsection{Variational Iteration Method}

As proposed by He [40], let us consider a generalised differential equation of the form

$L\{u(x)\}+N\{u(x)\}=f(x)$

with some prescribed constraints, where $u(x)$ is the function of interest, $L$ is a linear operator of the highest occurring derivative, $N$ is a nonlinear operator and $f$ is the source term.

The first call of the variational iteration method (VIM) is the construction of correction function for (11) as follow s: 


$$
u_{n+1}(x)=u_{n}(x)+\int_{0}^{x} \lambda(s)\left[L\left\{u_{n}(s)\right\}+\mathscr{N}\left\{u_{n}(s)\right\}\right.
$$

Where, $\lambda(s)$ is the general Lagrange multiplier which can be obtained via the variational theory, and $\mathbb{N}\left\{u_{n}(s)\right\}$ is the restricted term.

\subsubsection{Variational Iteration Orthogonal Collocation Method and Mamadu-Njoseh Polynomials}

Let

$$
S_{n}(t)=E_{n}(t)=I_{n}(t)=R_{n}(t)=\sum_{i=0}^{N} a_{i} \varphi_{i}(t) .
$$

be the approximate solution of the SEIR model with $a_{\mathrm{i}}$ "s being constants to be determined.

By the variational iteration method, we construct a correction functional for the system of equations

(9) as follows:

$$
\left\{\begin{array}{c}
S_{n+1}(t)=S_{n}(t)+\int_{0}^{t} \lambda_{1}(w)\left(\frac{d S_{n}}{d w}(w)+a \frac{S_{n}(w)}{N_{0}} I_{n}(w)+\frac{K}{N_{0}} S_{n}(w)-\left(\beta_{1}+\beta_{2}\right)+\left(a_{1}+a_{2}\right) \frac{S_{n}(w)}{w_{0}}+g N_{0}(w)-\tau S_{n}(w)\right) d w \\
E_{n+1}(t)=E_{n}(t)+\int_{0}^{t} \lambda_{2}(w)\left(\frac{d E_{n}}{d w}(w)-a \frac{S_{n}(w)}{N_{0}} I_{n}(w)-\frac{K}{N_{0}} S_{n}(w)+b E_{n}^{*}(w)+\left(a_{1}+a_{2}\right) \frac{E_{n}(w)}{N_{0}}+\rho E_{n}^{*}(w)+\tau E_{n}^{*}(w)\right) d w \\
I_{n+1}(t)=I_{n}(t)+\int_{0}^{t} \lambda_{n}(w)\left(\frac{d I_{n}}{d w}(w)-b E_{n}(w)+c I_{n}(w)+\left(a_{1}+a_{2}\right) \frac{I_{n}(w)}{N_{0}}+\tau I_{n}(w)\right) d w \\
R_{n+1}(t)(t)=R_{n}(t)+\int_{0}^{t} \lambda_{4}(w)\left(\frac{d R_{n}}{d w}(w)-c I_{n}(w)+\tau \mathbb{R}_{n}(w)-\rho E_{n}(w)\right) d w
\end{array}\right.
$$

where $\lambda_{i}(w), i=1(2) 4$, are the general Lagrange multipliers, $n \geq 0$, and $S_{n}(w), E_{n}(w), I_{n}(w)$ and $\tilde{R}_{n}(w)$ are restricted.

To estimate the values of $\lambda_{i}(w), i=1(2) 4$, we take the variation $\delta$ on both sides of (14) as follows:

$$
\left\{\begin{array}{l}
\delta S_{n+1}(t)=\delta S_{n}(t)+\delta \int_{0}^{t} \lambda_{1}(w)\left(\frac{d S_{n}}{d w}(w)+a \frac{S_{n}(w)}{N_{0}} I_{n}(w)+\frac{K}{N_{0}} S_{n}(w)-\left(\beta_{1}+\beta_{2}\right)+\left(a_{1}+a_{2}\right) \frac{S_{n}(w)}{N_{0}}+g \tilde{N}_{0}(w)-\tau S_{n}(w)\right) d w=0 \\
\delta E_{n+1}(t)=\delta E_{n}(t)+\delta \int_{0}^{t} \lambda_{2}(w)\left(\frac{d E_{n}}{d w}(w)-a \frac{S_{n}(w)}{N_{0}} I_{n}(w)-\frac{K}{N_{0}} S_{n}(w)+b E_{n}^{*}(w)+\left(a_{1}+a_{2}\right) \frac{E_{n}(w)}{N_{0}}+\rho \tilde{E}_{n}(w)+\tau \vec{E}_{n}^{*}(w)\right) d w=0(15) \\
\delta I_{n+1}(t)=\delta I_{n}(t)+\delta \int_{0}^{t} \lambda_{a}(w)\left(\frac{d I_{n}}{d w}(w)-b \vec{E}_{n}(w)+c I_{n}(w)+\left(a_{1}+a_{2}\right) \frac{I_{n}(w)}{N_{0}}+\tau I_{n}(w)\right) d w=0 \\
\delta R_{n+1}(t)(t)=\delta R_{n}(t)+\delta \int_{0}^{t} \lambda_{4}(w)\left(\frac{d R_{n}}{d w}(w)-c I_{n}(w)+\tau \tilde{R}_{n}(w)-\rho \vec{E}_{n}(w)\right) d w=0
\end{array}\right.
$$

Solving the above equation (15) we obtain the values 
$\lambda_{1}(w)=\lambda_{2}(w)=\lambda_{a}(w)=\lambda_{4}(w)=-1$.

Thus, equation (14) becomes

$$
\left\{\begin{array}{l}
S_{n+1}(t)=S_{n}(t)-\int_{0}^{t}\left(\frac{d S_{n}}{d w}(w)+a \frac{S_{n}(w)}{N_{0}} I_{n}(w)+\frac{K}{N_{0}} S_{n}(w)-\left(\beta_{1}+\beta_{2}\right)+\left(\alpha_{1}+\alpha_{2}\right) \frac{S_{n}(w)}{N_{0}}+g N_{0}(w)-\tau S_{n}(w)\right) d w \\
E_{n+1}(t)=E_{n}(t)-\int_{0}^{t}\left(\frac{d E_{n}}{d w}(w)-a \frac{S_{n}(w)}{N_{0}} E_{n}(w)-\frac{K}{N_{0}} S_{n}(w)+b E_{n}(w)+\left(\alpha_{1}+a_{2}\right) \frac{E_{n}(w)}{N_{0}}+\rho E_{n}(w)+\tau E_{n}(w)\right) d w \\
I_{n+1}(t)=I_{n}(t)-\int_{0}^{t}\left(\frac{d I_{n}}{d w}(w)-b E_{n}(w)+c I_{n}(w)+\left(a_{1}+a_{2}\right) \frac{I_{n}(w)}{N_{0}}+\tau I_{n}(w)\right) d w \\
R_{n+1}(t)(t)=R_{n}(t)-\int_{0}^{t}\left(\frac{d R_{n}}{d w}(w)-c I_{n}(w)+\tau R_{n}(w)-\rho E_{n}(w)\right) d w
\end{array}\right.
$$

To implement the iterative (16), we require an initial approximation to kick-off the iteration process which we shall obtain from using equation (10) and (14) as given below .

$$
\left\{\begin{array}{r}
S_{0}(t)=\sum_{i=0}^{W} a_{i} \varphi_{i}(t)-K_{1} \\
E_{0}(t)=\sum_{i=0}^{W} a_{i} \varphi_{i}(t)-K_{2} \\
I_{0}(t)=\sum_{i=0}^{W} a_{i} \varphi_{i}(t)-K_{a} \\
R_{0}(t)=\sum_{i=0}^{W} a_{i} \varphi_{i}(t)-K_{4}
\end{array}\right.
$$

Consequently, for $n \geq 0$, the required approximate solutions resulting from using (16) and (17) is thus given as the series solution

$$
\left\{\begin{array}{r}
S(t)=\sum_{i=0}^{N} S_{i} \\
E(t)=\sum_{i=0}^{N} E_{i} \\
I(t)=\sum_{i=0}^{N} I_{i} \\
R(t)=\sum_{i=0}^{N} I_{i}
\end{array}\right.
$$

where the values of $a_{i}$ 's are computed via orthogonal collocation after linearization.

\section{Results}

Now, implementing the above iterative procedures for $n \geq 0$ with the aid of MAPLE software for $N=3$ with the values $K_{1}=2500, K_{2}=1, K_{3}=1, K_{4}=0$, we obtain the following approximations arising from equation (16) and (17) respectively:

Now, implementing the above iterative procedures for $n \geq 0$ with the aid of MAPLE software for $N=3$ with the values $K_{1}=2500, K_{2}=1, K_{a}=1, K_{4}=0$, we obtain the following approximations arising from equations (16) - (18) respectively: 


$$
\begin{aligned}
& S(t):=2 a_{0}+20 a_{1}+332 a_{2}+5564 a_{3}-5000 \\
& -\frac{a \beta\left(a_{0}+10 a_{1}+166 a_{2}+2782 a_{3}-2500\right)\left(a_{0}+10 a_{1}+166 a_{2}+2782 a_{3}-1\right) t}{N_{0}} \\
& -\frac{K\left(a_{0}+10 a_{1}+166 a_{2}+2782 a_{3}-2500\right) t}{N_{0}}+\beta_{1} t+\beta_{E} t-\left(\frac{\alpha_{1}}{N_{0}}+\frac{\alpha_{E}}{N_{0}}\right)\left(a_{0}\right. \\
& \left.+10 a_{1}+166 a_{2}+2782 a_{3}-2500\right) t+g N_{0} t-\tau\left(a_{0}+10 a_{1}+166 a_{2}+2782 a_{3}\right. \\
& -2500) t \\
& E(t):=2 a_{0}+20 a_{1}+332 a_{2}+5564 a_{3}-2 \\
& +\frac{a \beta\left(a_{0}+10 a_{1}+166 a_{2}+2782 a_{3}-2500\right)\left(a_{0}+10 a_{1}+166 a_{2}+2782 a_{3}-1\right) t}{N_{0}} \\
& +\frac{K\left(a_{0}+10 a_{1}+166 a_{2}+2782 a_{3}-2500\right) t}{N_{0}}-\left(b+\frac{\alpha_{1}}{N}+\frac{\alpha_{E}}{N}+\rho+\tau\right)\left(a_{0}\right. \\
& \left.+10 a_{1}+166 a_{2}+2782 a_{3}-1\right) t \\
& I(t):=3 a_{0}+30 a_{1}+498 a_{2}+8346 a_{3}-3+2 b\left(a_{0}+10 a_{1}+166 a_{2}+2782 a_{3}-1\right) t \\
& -2\left(c+\frac{\alpha_{1}}{N_{0}}+\frac{\alpha_{E}}{N_{0}}+\tau\right)\left(a_{0}+10 a_{1}+166 a_{2}+2782 a_{3}-1\right) t+b\left(a_{0}+10 a_{1}\right. \\
& +166 a_{2}+2782 a_{3}-1 \\
& +\frac{a \beta\left(a_{0}+10 a_{1}+166 a_{2}+2782 a_{3}-2500\right)\left(a_{0}+10 a_{1}+166 a_{2}+2782 a_{3}-1\right) t}{N_{0}} \\
& +\frac{K\left(a_{0}+10 a_{1}+166 a_{2}+2782 a_{3}-2500\right) t}{N_{0}}-\left(b+\frac{\alpha_{1}}{N}+\frac{\alpha_{E}}{N}+\rho+\tau\right)\left(a_{0}\right. \\
& \left.\left.+10 a_{1}+166 a_{2}+2782 a_{3}-1\right) t\right) t-\left(c+\frac{\alpha_{1}}{N_{0}}+\frac{\alpha_{E}}{N_{0}}+\tau\right)\left(a_{0}+10 a_{1}+166 a_{2}\right. \\
& +2782 a_{3}-1+b\left(a_{0}+10 a_{1}+166 a_{2}+2782 a_{3}-1\right) t-\left(c+\frac{\alpha_{1}}{N_{0}}+\frac{\alpha_{E}}{N_{0}}+\tau\right)\left(a_{0}\right. \\
& \left.\left.+10 a_{1}+166 a_{2}+2782 a_{3}-1\right) t\right) t
\end{aligned}
$$




$$
\begin{aligned}
R(t) & :=3 a_{0}+30 a_{1}+498 a_{2}+8346 a_{3}+2 c\left(a_{0}+10 a_{1}+166 a_{2}+2782 a_{3}-1\right) t \\
& -2 \tau\left(a_{0}+10 a_{1}+166 a_{2}+2782 a_{3}\right) t+2 \rho\left(a_{0}+10 a_{1}+166 a_{2}+2782 a_{3}-1\right) t \\
& +c\left(a_{0}+10 a_{1}+166 a_{2}+2782 a_{3}-1+b\left(a_{0}+10 a_{1}+166 a_{2}+2782 a_{3}-1\right) t\right. \\
& \left.-\left(c+\frac{\alpha_{1}}{N_{0}}+\frac{\alpha_{E}}{N_{0}}+\tau\right)\left(a_{0}+10 a_{1}+166 a_{2}+2782 a_{3}-1\right) t\right) t-\tau\left(a_{0}+10 a_{1}\right. \\
& +166 a_{2}+2782 a_{3}+c\left(a_{0}+10 a_{1}+166 a_{2}+2782 a_{3}-1\right) t-\tau\left(a_{0}+10 a_{1}+166 a_{2}\right. \\
& \left.\left.+2782 a_{3}\right) t+\rho\left(a_{0}+10 a_{1}+166 a_{2}+2782 a_{3}-1\right) t\right) t+\rho\left(a_{0}+10 a_{1}+166 a_{2}\right. \\
& +2782 a_{3}-1 \\
+ & \frac{a \beta\left(a_{0}+10 a_{1}+166 a_{2}+2782 a_{3}-2500\right)\left(a_{0}+10 a_{1}+166 a_{2}+2782 a_{3}-1\right) t}{N_{0}} \\
+ & \frac{K\left(a_{0}+10 a_{1}+166 a_{2}+2782 a_{3}-2500\right) t}{N_{0}}-\left(b+\frac{\alpha_{1}}{N}+\frac{\alpha_{E}}{N}+\rho+\tau\right)\left(a_{0}\right. \\
+ & \left.\left.10 a_{1}+166 a_{2}+2782 a_{3}-1\right) t\right) t \quad(b)
\end{aligned}
$$

Using the assumed values,

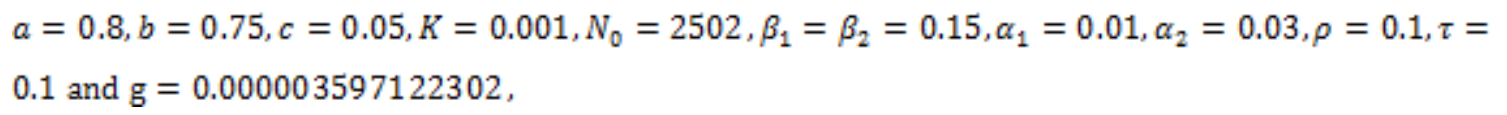

on the above equations and collocating orthogonally after linearization for the values of the

unknowns $a_{i \mathrm{i}}^{i} s_{x}$ and substituting back into the (18) yields us the following approximations:

$$
\begin{aligned}
S(t) & :=2500-25.53132774 \cdot t-0.143869926 \cdot t^{2}+0.013851166 \cdot t^{3}+0.00002273066243 \cdot t^{4} \\
& -4.79158897410^{-7} t^{5}+4.31004739710^{-11} t^{6} \\
E(t) & :=1-0.059656274 \cdot t+0.29733881 \cdot t^{2}-0.09860941 \cdot \mathrm{t}^{3}+4.8381106310^{-4} \cdot \mathrm{t}^{4} \\
& -4.79158897410^{-7} \cdot \mathrm{t}^{5}+4.31004739710^{-11} t^{6} \\
I(t) & :=1+0.689984012 \cdot t-0.043076138 \cdot t^{2}+0.074410709 \cdot t^{3} \\
R(t) & :=0.15 \cdot t+0.013516786 \cdot t^{2}+9.1483020810^{-3} \cdot \mathrm{t}^{3}
\end{aligned}
$$

3.2. Figures, Tables and Schemes 


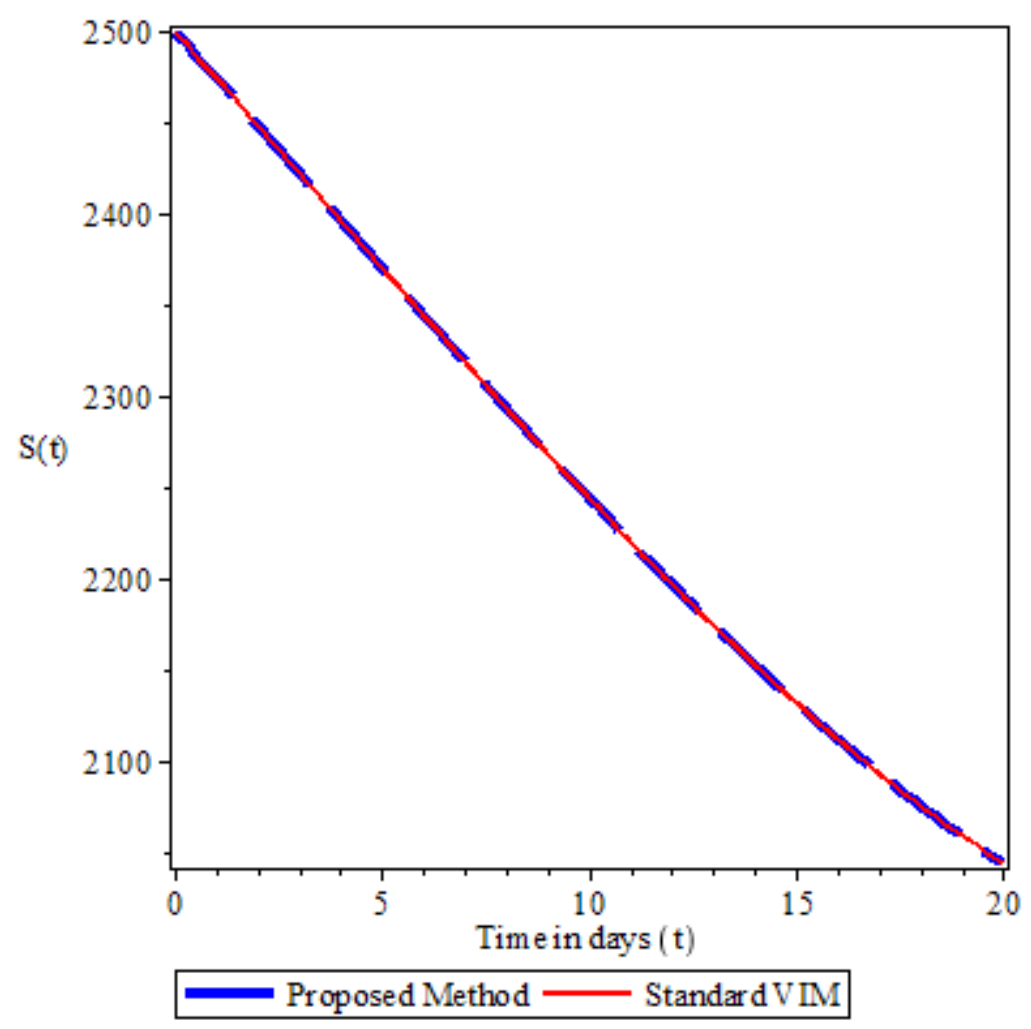

Figure 3: Comparison of the VIOCM and standard VIM of Susceptible individuals in the population against time $(t)$.

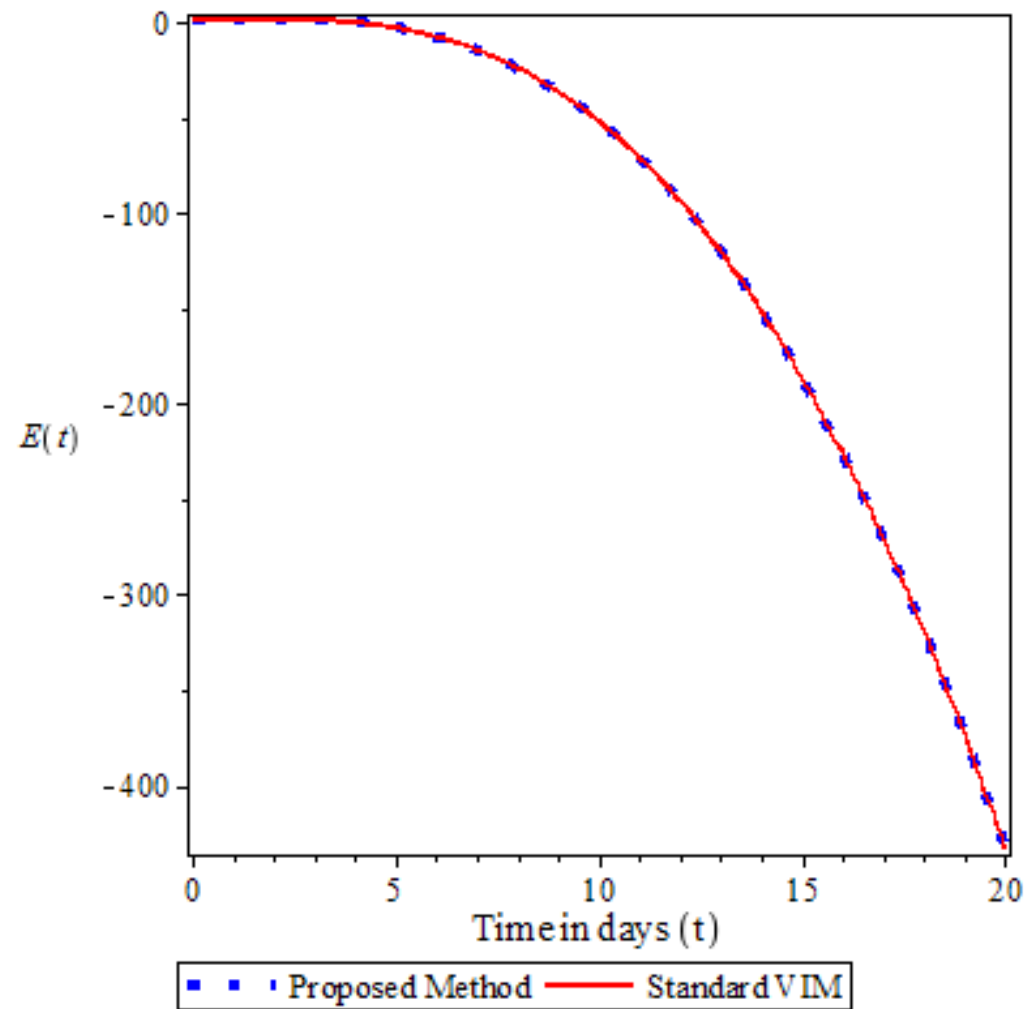

Figure 4: Comparison of the VIOCM and standard VIM of Exposed individuals in the population against time $(t)$. 


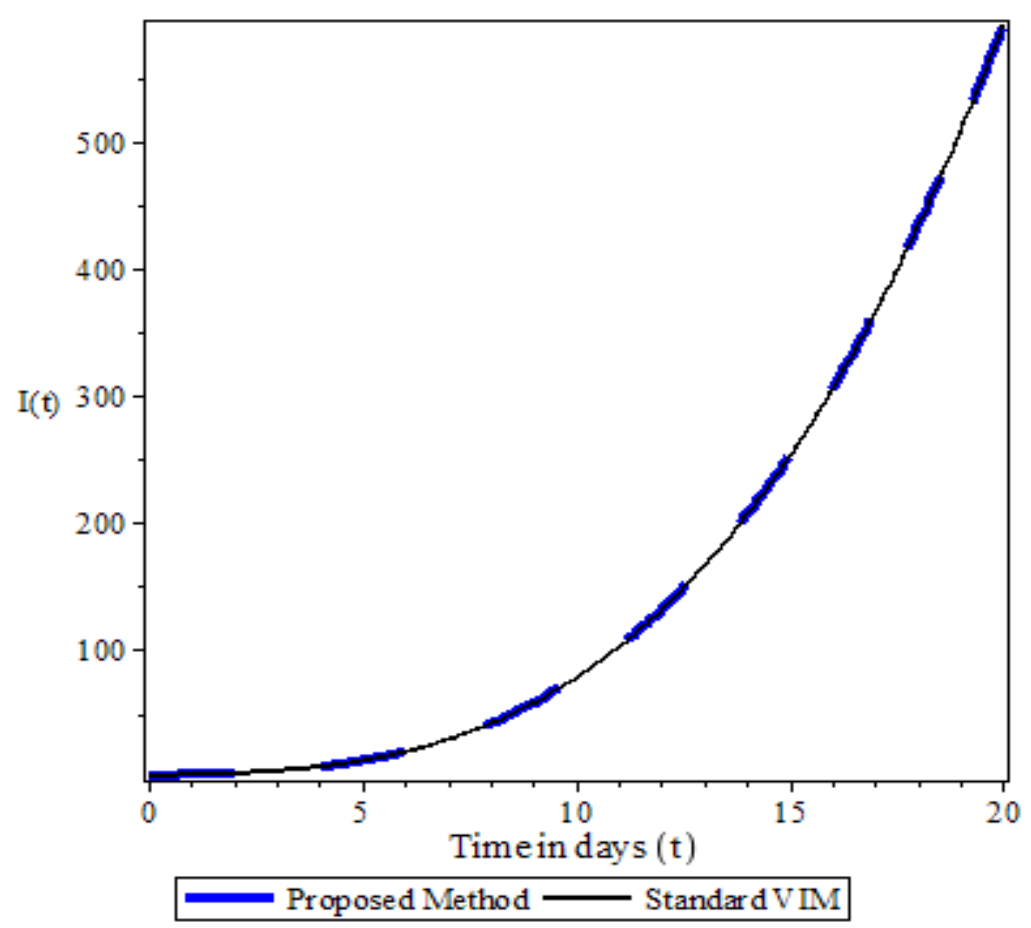

Figure 5: Comparison of the VIOCM and standard VIM of Infected individuals in the population against time $(t)$.

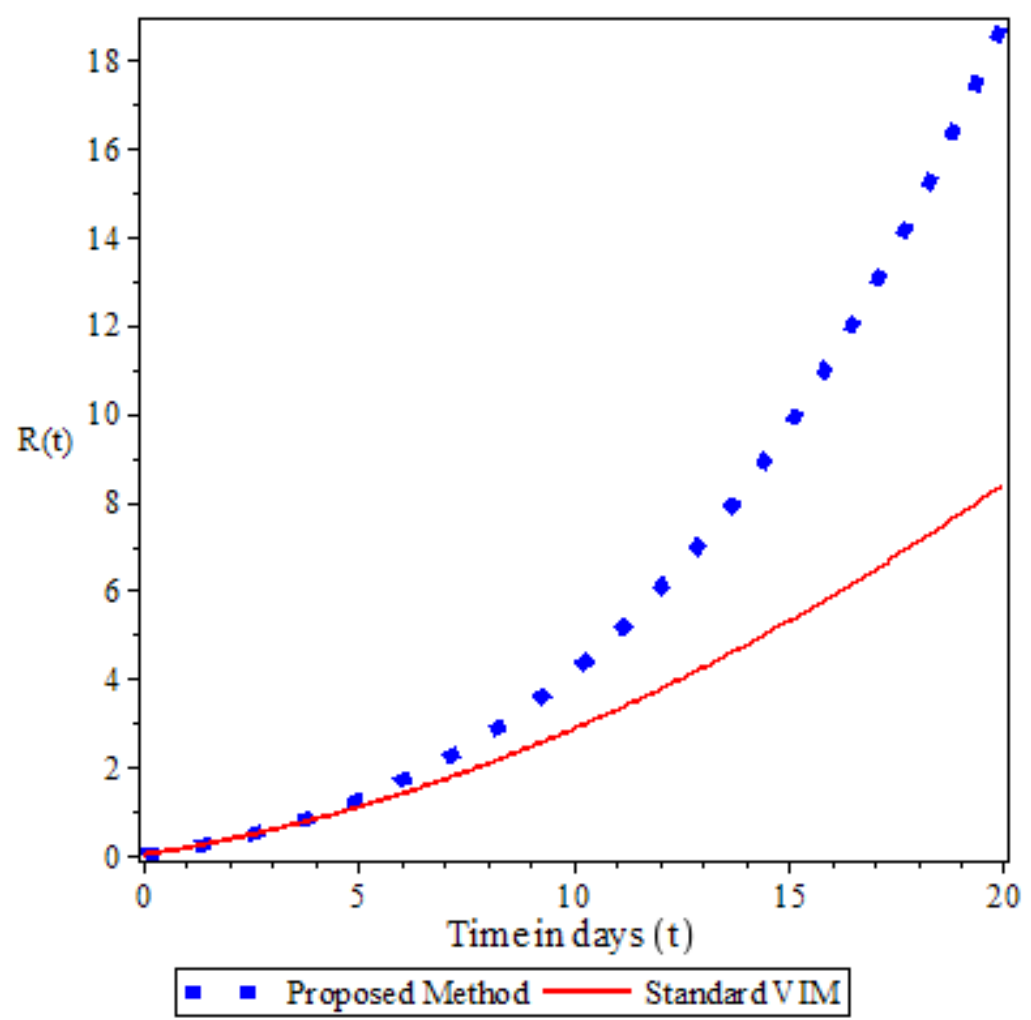

Figure 6: Comparison of the VIOCM and standard VIM of Recovered individuals in the population against time $(t)$. 
Table 1: Percentage decline of susceptible individuals recorded against time in days $(t)$.

\begin{tabular}{|l|l|l|l|l|l|l|l|l|l|l|l|}
\hline Susceptible (\%) & 99.9 & 97.9 & 95.9 & 93.9 & 91.9 & 89.9 & 87.9 & 85.9 & 81.9 & 83.9 & 79.9 \\
\hline Time in days $(t)$ & 0 & 2 & 4 & 6 & 8 & 10 & 12 & 14 & 16 & 18 & 20 \\
\hline
\end{tabular}

Table 2: Percentage of Exposed individuals recorded against time in days $(t)$.

\begin{tabular}{|l|l|l|l|l|l|l|l|l|l|l|l|}
\hline Exposed (\%) & 0 & 0 & 0 & -0.4 & -0.8 & -2 & -4 & -6 & -9 & -13 & -20 \\
\hline Time in days & 0 & 2 & 4 & 6 & 8 & 10 & 12 & 14 & 16 & 18 & 20 \\
$(t)$ & & & & & & & & & & & \\
\hline
\end{tabular}

Table 3: Percentage increase of infected individuals recorded against time in days $(t)$.

\begin{tabular}{|l|l|l|l|l|l|l|l|l|l|l|l|}
\hline Infectious (\%) & 0 & 0 & 0.4 & 1.2 & 2 & 3.2 & 5.6 & 8 & 12 & 17.99 & 23.98 \\
\hline Time in days & 0 & 2 & 4 & 6 & 8 & 10 & 12 & 14 & 16 & 18 & 20 \\
$(t)$ & & & & & & & & & & & \\
\hline
\end{tabular}

Table 4: Percentage of recovered individuals recorded against time in days $(t)$.

\begin{tabular}{|l|l|l|l|l|l|l|l|l|l|l|l|}
\hline $\begin{array}{l}\text { Recovered } \\
(\%)\end{array}$ & 0 & 0.008 & 0.04 & 0.07 & 0.1 & 0.2 & 0.24 & 0.32 & 0.41 & 0.56 & 0.74 \\
\hline $\begin{array}{l}\text { Time in } \\
\text { days }(t)\end{array}$ & 0 & 2 & 4 & 6 & 8 & 10 & 12 & 14 & 16 & 18 & 20 \\
\hline
\end{tabular}

\section{Discussion}

Haven implemented the proposed method for the numerical approximation of the SEIR Coronavirus model, the following observations are recorded:

i. There is decline of susceptible individuals in the population with a common difference of 2 as shown in Figure 3. For instance, at the initial time (in days), $99.9 \%$ of the population is susceptible to the COVID-19 disease. In time (in days) 20, $79.9 \%$ of the population is noticed to be susceptible to the disease. Table 1 shows the percentage decline of susceptible individuals recorded against time in days $(t)$.

The reason for such a decline could be as a result of the following factors: a properly functioning immune system, age factor, genetics factor, environmental factor, nutrition and by chance [48]. 
ii. Individuals were less exposed individuals to the COVID-19 disease in the first 20 days (in time) in the population as shown in Figure 4. Table 2 shows the percentage of exposed individuals recorded against time in days $(t)$.

iii. There is a rapid rate of infected individuals to the COVID-19 disease in the population as shown in Figure 5. Table 3 shows the percentage increase of infected individuals recorded against time in days ( $(t)$. This rapid increase is caused either by coming in contact with the infected host or through contaminated objects or surfaces [49].

iv. Recovered individuals to the COVID-19 disease in the population is notable as shown in Figure 6.

Table 4 show s the percentage of recovered individuals recorded against time in days $(t)$.

\section{Conclusions}

Since the outbreak of the deadly novel coronavirus (COVID-19) epidemic in Wuhan, China, many mathematical models have been proposed to study the various compartments of the SEIR in the transmission among individuals in the population. Consequently, the SEIR model has been solved either analytically or numerically by the emerging researchers since the outbreak. How ever, the use of orthogonal polynomials has never been adopted in the approximation of the analytic solution of the SEIR model. In this article, we have successfully implemented the use of orthogonal Mamadu-Njoseh polynomials via the proposed method which is the variational iteration orthogonal collocation method to seek the solution of the SEIR model. It was observed that the proposed method converges rapidly to the exact solution even as $N$ increases. This suggests that the use of orthogonal polynomials as trial functions for the SEIR model is indeed an effective approximant as it produces the analytic solution at just few iterations. Resulting numerical evidences were compared with the standard variational iteration method as available in the literature [38] as shown in the Figures 3 - 6.

Author Contributions: Conceptualization, Ebimene J. Mamadu and Ignatius N. Njoseh; Formal analysis, Ebimene J. Mamadu; Funding acquisition, Ignatius N. Njoseh; Investigation, Ebimene J. Mamadu and Ignatius N. Njoseh; Methodology, Ebimene J. Mamadu and Ignatius N. Njoseh; Project administration, Ignatius N. Njoseh; Resources, Newton I. Okposo; Software, Ebimene J. Mamadu; Supervision, Ignatius N. Njoseh; Validation, Ignatius N. Njoseh; Writing - original draft, Ebimene J. Mamadu and Ignatius N. Njoseh; Writing-review \& editing, Ignatius N. Njoseh.

Funding: Please add: "This research received no external funding.

Acknowledgments: Not applicable.

\section{Conflicts of Interest: Declaration of interests}

The authors declare that they have no known competing financial interests or personal relationships that could have appeared to influence the w ork reported in this paper.

The authors declare that there w as no financial interests/personal relationships which may be considered as potential competing interests. 


\section{References}

[1] Hui, D., Azhar, E., Madani, T., Ntoumi, F., Kock, R., Dar, O., Ippolito, G., Mchugh, T., Memish, Z., Drosten, C., Zumla, A., and Petersen, E. (2020). The Continuing epidemic threat of novel coronaviruses to global health - the latest novel coronavirus outbreak in Wuhang, China, International Journal of Infectious Diseases, 10.1016/j.ijid.2020.01.009.

[2] 1mg, “Coronavirus No-Panic Help guide." Retrieved on 24/04/2020. https://smef.org.uk/w p-content/uploads/2020/03/Corona-Ebook.pdf.pdf.pdf.

[3] Tang, B., Bragazzi, N. L., Li, Q., Tang, S., Xiao, Y. and Wu, J. (2020). An Updated Estimation of the Risk of Transmission of the Novel Coronavirus (2019-nCov). Infectious Disease Modelling, 5:248-255.

[4] World Health Organisation (2020). Coronavirus Disease (COVID-19). Situation Reports 1-45.https://w w w.who.int/emergencies/diseases/novel-coronavirus-2019/situation-reports.

[5] Gorbalenya, A. E. et al (2020). The species severe acute respiratory syndrome-related coronavirus: classifying 2019-nCoV and naming it SARS-CoV-2, Nature Microbiology. DOI: 10.1038/s41564-020-0695-z.

[6] Kupferschmidt, K., Cohen, J. (2020). Will novel virus go pandemic or be contained? Science, 367 (6478), $610-611$.

[7] Coronavirus is now expected to curb global economic growth by 0.3\% in 2020 . https://www .forbes.com/sites/sergeiklebnikov/2020/02/11/coronavirus-is-now-expected-to-c urb-global-economic-grow th-by-03-in-2020/\#5de149ad16da.

[8] Su, S., Wong, G., Shi, W., Liu, J., Lai, A. C. K., Zhou, J., Liu, W. Bi, Y., and Gao, G. F. (2016). Epidemiology, genetic recombination, and pathogenesis of coronaviruses, Trends Microbiol, $24(6), 490-502$.

[9] W. Ji, W. Wang, X. Zhao, J. Zai, and X. Li (2020). “Homologous recombination within the spike glycoprotein of the newly identified coronavirus may boost cross species tr ansmission from snake to human, Journal of Medical Virology, vol. 92, no. 4, 2020.

[10] Madubueze, C. E., Kimbir, A. R. and Aboiyar, T. (2018). Global Stability of Ebola Vir us Disease Model with Contact Tracing and Quarantine, Appl. Appl. Math., 13(1): 383 - 403.

[11] Kim, Y., Lee, C., Chu, S. Choe, S., Hong, S. and Shin, Y. (2016). The Characteristics of Middle Eastern Respiratory Syndrome Coronavirus Transmission dynamics in South Korea, Osong Public Health and Research Perspectives, 7(1): 49-55.

[12] Li, Q., Guan, X., Wu, P., Wang, X., Zhou, L., Tong, Y. (2020). Early Transmission Dynamics in Wuhan, China, of Novel Coronavirus-Infected Pneumonia, The New England Journal of Medicine, 382(13): 1199-1207.

[13] Wu, J. T., Leung, K. and Leung, G. M. (2020). Nowcasting and Forecasting the Potential Domestic and International Spread of the 2019-nCoV Outbreak Originating in Wuhan, China: a modelling study. THE LANCET, 395 (10225): 689 - 697.

[14] Zhao, S., Lin, Q., Ran, J., Musa, S. S., Yang, G. and Wang, W. (2020). Preliminary Estimation of the Basic Reproduction Number of Novel Coronavirus (2019-nCoV) in China, from 2019 
to 2020: A Data-Driven Analysis in the Early Phase of the Outbreak. Int J Infect Dis. 92: 214 217.

[15] Zhao, S., Musa S. S., Lin, Q., Ran, J., Yang, G. and Wang, W. (2020). Estimating the Unreported Number of novel Coronavirus (2019-nCoV) Cases in China in the First half of January 2020: A Data-Driven Modelling Analysis of the Early Outbreak.

[16] Harir, A., Melliani, S., El Harfi, H., and Chadli, L. S. (2020). Variational Iteration Method and Differential Transformation Methodfor Solving the SEIR Epidemic Model, International Journal of Differential Equations. https://doi.org/10.1155/2020/3521936.

[17] Chinwendu E. M., Sambo D. and Isaac O. O. (2020). Controlling the Spread of COVID-19: Optimal Control Analysis. medRxiv preprint doi: ttps://doi.org/10.1101/2020.06.08.20125393.

[18] Gumel, A. B., Ruan, S., Day, T., Watmough, J., Brauer, F., Driessche van den, P., Gabrielson, D., Bow man, C., Alexander, E., Ardal, S., Wu, J., and Sahai, B. M. (2004), Modelling Strategies for Controlling SARS Outbreaks. Proc. R. Soc. Lond. B, 271: 2223- 2232.

[19] Getachew, T. (2017). Mathematical Model for Co-Infection of Pneumonia and Typhoid Fever Disease with Optimal Control, Pan African University.

[20] Elzaki, T.M. and Ezaki, S.M. (2011) On the Elzaki transform and ordinary differential equation with variable coefficients, Advances in Theoretical and Applied mathematics ,6: 41-46.

[21] Elzaki, T.M. and Ezaki, S.M. (2011). Application of new transform "Elzaki transform" to partial differential equations, Global Journal of Pure and Applied Mathematics, 7:65-70.

[22] Mandelzweig, V.B. and Tabakin, F. (2011). Quasilinearization approach to nonlinear problems in physics with application to nonlinear ODEs, Comput. Phys. Commun. 141:268-281.

[24] Ramos, J.I. (2003). Linearization method in classical and quantum mechanics, Comput. Phys. Commum, 153:199-208.

[25] Inokuti, M., Sekine, H., and Mura, T. (1978). General use of the Lagrange multiplier in nonlinear mathematical physics, in Variational Method in the Mechanics of Solids, $S$. Nemat-Nassed, Ed., Pergemon Press, Oxford, UK, 156-162.

[26] Chen, T.M. Rui, J., Wang, Q. P., Zhao, Z. Y., Cui, J. A. and Yin, L. (2020). A mathematical model for simulating the phase-based transmissibility of a novel coronavirus, Infectious Diseases of Poverty, (9)24.

[27] Kanth, S. V. and Aruna, K. (2009). Two-dimensional differential transform method for solving linear and non-linear Schrodinger equations, Chaos, Solitons $\mathcal{E}$ Fractals, 41(5):2277-2281, 2009.

[28] Shah, K., Alqudah, M. A., Jarad, F., and Abdeljawad, T. (2020). Semi analytical study of Pine Wilt Disease model with convex rate under Caputo-Febrizio fractional order derivative, Chaos, Solitons E Fractals, 135, Article ID 109754, 2020.

[29] Mamadu, E.J. and Njoseh, I.N. (2017). Elzaki transform method for delay differential equation, Boson Journal of Modern Physics, 3(1):2454-8413

[30] Ogunfiditimi, F. O. (2015). Numerical solution of delay differential equations using Adomian decomposition method, The International Journal of Engineering and Science, 4(5):18 $-23$.

[31] Mirzaee, F. and Latifi, L. (2011). Numerical solution of delay differential equations by differential transform method, J. Sci. I. A. U (JSIAU), 20(78/2): 83-88. 
[32] Ismail, F. Al-Khasawneh, A.R, Lwin, A.S and Suleiman, M. (2002). Numerical treatment of delay differential equations by Runge-Kutta method using Hermite interpolation, Mathematika, 18(2):79-90

[33] Kuma, D. and Kadalbajoo, M.K. (2012). Numerical treatment of singularly perturbed delay differential equations using B-spline collocation method on Shishkin mesh, Joumal of Numerical Analysis, Industrial and Applied Mathematics (JNAIAM), 7(3-4):73-90.

[34] Njoseh, I.N. and Mamadu, E.J. (2016). Numerical solutions of generalized Nth order boundary value problems using power series approximation method, Applied Mathematics, 7:1215-1224. http://dx.doi.org/10.4236/am.2016.711107.

[35] Zennaro, M. (1985). On the P-stability of one-step collocation for delay differential equations, International Series of Numerical Mathematics (ISNM), 74:334-343.

[36] Njoseh, I.N. and Mamadu, E.J. (2016). Numerical solutions of fifth order boundary value problems using Mamadu-Njoseh polynomials. Science World Journals, 11(4): 21-24

[37] Biswas, M.H.A., Paiva, L.T. and MdR de Pinho. (2014). SEIR model for control of infectious diseases with constraints, Mathematical Biosciences and Engineering, 11(4): 761-784

[38] Harir, A., Melliani, S., El Harfi, H. and Chadli, L. S. (2020). Variational iteration method and differential transformation method for solving the SEIR epidemic model. International Journal of Differential Equations, Article ID 3521936, 7 pages, https://doi.org/10.1155/2020/3521936

[39] Abbasbandy, S. and Shivanian, E. (2009). Application of the variational iteration method for system of nonlinear Volterra's integro-differential equations, Mathematics and Computational Applications, 14(2):147-158.

[40] He, J.H. (2006). Some asymptotic methods for strongly nonlinear equations, International Journal of Modern Physics B, 20(10):1141-1199.

[41] Brunner, H. (2004). Collocation methods for Volterra integral and related functional equations, Cambridge University Press, Cambridge CB2 2RU, UK.

[42] Olagunju, A.S. and Joseph, F.L. (2013). Third kind Chebychev Polynomials in Collocation Methods of Solving Boundary Value Problems. IOSR Journal of Mathematics, 7(2):42-47.

[43] Ji, W., Wang, W., Zhao, X., Zai, J. and Li, X. (2020). Homologous recombination within the spike glycoprotein of the newly identified coronavirus may boost cross-species transmission from snake to human, Journal of Medical Virology, 92(4).

[44] Abdo, M. S. Shah, K., Wahash, H. A. and S. K. Panchal, (2020). On a comprehensive model of the novel coronavirus (COVID-19) under Mittag-Leffler derivative, Chaos, Solitons Fractals, 135, 109867.

[45] Njoseh I.N and Mamadu E.J (2016) the numerical solution of fifth-order value problems using Mamadu-Njoseh polynomials, Science World Journal, 11(4):21-24.

[46] Mamadu, E.J. and Njoseh, I.N. (2016). Numerical solutions of Volterra equations using Galerkin method with certain orthogonal polynomials. Journal of Applied Mathematics and Physics, 4:376-382.

[47] Mohammed, D.S. (2014). Numerical solution of fractional integro-differential equations by least squares method and shifted Chebyshev polynomial, Mathematical Problems in Engineering, 2014:1-5 Article ID 431965 
[48] Chapman, S., Hill, A. (2012) Human genetic susceptibility to infectious disease. Nat Rev Genet 13, 175-188. https://doi.org/10.1038/nrg3114

[49] Michael S., Shanna R., Jason Y., Gregory W., Wade W., Brian G., David M., Melissa K., Katie B., Stew art W., Brian H., Jordan B., Denise F., Idris H., Jennifer B., Louis A., Victoria W., Michael H., Paul D. (2020) AirborneSARS-CoV-2 is rapidly inactivated by simulated sunlight, The Journal of Infectious Diseases, 222(4):564-571, https://doi.org/10.1093/infdis/jiaa334 